TRANSACTIONS OF THE

AMERICAN MATHEMATICAL SOCIETY

Volume 358, Number 2, Pages 473-489

S 0002-9947(05)04024-9

Article electronically published on September 26, 2005

\title{
ON MEROMORPHIC FUNCTIONS WITH FINITE LOGARITHMIC ORDER
}

\author{
PETER TIEN-YU CHERN
}

\begin{abstract}
By using a slow growth scale, the logarithmic order, with which to measure the growth of functions, we obtain basic results on the value distribution of a class of meromorphic functions of zero order.
\end{abstract}

\section{INTRODUCTION AND RESULTS}

The theory of meromorphic functions of finite positive order is fairly complete as compared to the theory of functions of order zero. Techniques that work well for functions of finite positive order often do not work for functions of order zero. In order to make some progress with functions of order zero, we make use of the concept of logarithmic order (see [C1], [C2]) to develop our results. An increasing function $\phi(r)$ is said to be of logarithmic order $\lambda$ if

$$
\limsup _{r \rightarrow+\infty} \frac{\log \phi(r)}{\log \log r}=\lambda \text {. }
$$

In section 1, we develop in Theorem 1.1 an integral characterization for increasing functions with finite logarithmic order. In section 2, we define the logarithmic order of a meromorphic function $f$ to be the logarithmic order of its characteristic function $T(r, f)$, and we develop some properties for meromorphic functions with finite positive logarithmic order. In section 3 , we show that for a meromorphic function $f$ and a complex value $a$, if the logarithmic order of $n(r, f=a)$ is $\lambda_{\log }(a)$, then the logarithmic exponent of convergence of the $a$-points equals the logarithmic order. In section 4 we show that $N(r, f=a)$ is of logarithmic order $1+\lambda_{\log }(a)$ (Theorem 4.1).

For a meromorphic function $f$, a complex value $a$ is called a Borel exceptional value if the order of $n(r, f=a)$ is less than the order of $f$. Very little is known about the Borel exceptional value(s) for meromorphic functions of zero order. A complex value $a$ is called a logarithmic Borel exceptional value if $\lambda_{\log }(a)$, the logarithmic order of $n(r, f=a)$, is less than $\lambda-1$, where $\lambda$ is the logarithmic order of $f$. Further, a complex value $a$ is called a reduced logarithmic Borel exceptional value if the logarithmic order of the function $\bar{n}(r, f=a)$, the number of distinct roots of

Received by the editors March 11, 2003.

2000 Mathematics Subject Classification. Primary 30D30, 30D35.

Key words and phrases. Logarithmic Borel exceptional value, Borel direction of logarithmic order, finite logarithmic order.

This paper was supported in part by the NSC R.O.C. under the grants NSC 86-2115-M214001 and NSC 93-2115-M-214-005, a fund from Academia Sinica (Taipei, Taiwan), and funds from Michigan State University and Northern Illinois University. 
the equation $f(z)=a$ in the disc $|z| \leq r$, is less than $\lambda-1$ (where $\lambda$ again is the logarithmic order of $f$ ). In section 5 , we prove that any non-constant meromorphic function of finite logarithmic order has at most two logarithmic Borel exceptional values (Theorem 5.1). This result adds information about an assertion on Borel exceptional values by M. L. Cartwright [Ca, p. 24]. In section 6, we develop some results about derivatives of meromorphic functions with finite logarithmic order and, in Theorem 6.3, relate these results to Borel exceptional values for a combination of the function itself and a specified derivative of the function. In section 7 , we show that a non-constant meromorphic function with finite logarithmic order can have at most one logarithmic Borel exceptional value, and that if the function is an entire function, then it has no finite logarithmic exceptional values (Theorem 7.2). We also show that that there are infinitely many transcendental entire functions of logarithmic order one (Theorem 7.3), and that for each positive number $\lambda>1$ there exists an entire function of logarithmic order $\lambda$ (Theorem 7.4), and for a transcendental meromorphic function $f, T(r, f)$ is usually dominated by three integrated counting functions; however, when $f$ is of finite logarithmic order, $T(r, f)$ can be dominated by two integrated counting functions (Theorem 7.1).

In section 8 , we prove that if $f$ is a transcendental entire function with finite logarithmic order $\lambda$, if its lower logarithmic order

$$
\mu=\liminf _{r \rightarrow+\infty} \frac{\log T(r, f)}{\log \log r}
$$

satisfies the inequality $\lambda<\mu+1$, and if $c$ is a constant satisfying $0 \leq c<\lambda-\mu$, then for each finite complex value $a$, we have the asymptotic equivalences

$$
\begin{gathered}
N(r, f=a) \sim N\left(r(\log r)^{c}, f\right) \sim T(r, f) \sim T\left(r(\log r)^{c}, f\right) \\
\sim \log M(r, f) \sim \log M\left(r(\log r)^{c}, f\right) .
\end{gathered}
$$

This improves a result of Jianwu Sun $[\mathrm{Su}]$.

For a meromorphic function, let $n(r, \theta, \epsilon, f=a)$ denote the number of roots of the equation $f(z)=a$ in the set $\{z:|z| \leq r, \theta-\epsilon<\arg z<\theta+\epsilon\}$, where $a$ is a complex value, and $\theta, \epsilon$, and $r$ are real numbers with $\epsilon>0$ and $r \geq 0$. If $f$ is a meromorphic function with finite logarithmic order $\lambda$, then the ray $\Delta(\theta)=\{z: \arg z=\theta\}$ is called a Borel direction of logarithmic order $\lambda-1$ if

$$
\limsup _{r \rightarrow+\infty} \frac{\log n(r, \theta, \epsilon, f=a)}{\log \log r}=\lambda-1
$$

holds for each small positive number $\epsilon$ and for every complex number $a$ (including $\infty)$, with at most two possible exceptions. In [C3, Theorem 2], we prove that a meromorphic function with finite logarighmic order $\lambda$ and satisfying the growth condition

$$
\limsup _{r \rightarrow+\infty} \frac{T(r, f)}{(\log r)^{2}}=+\infty
$$

has a Borel direction of logarithmic order $\lambda-1$ (Theorem 9.1). In a private communication, Seng-Jian $\mathrm{Wu}$ asked the following question: if $f$ is a meromorphic function with finite logarithmic order $\lambda$, does there exist a real number $\theta$ such that for any small positive number $\epsilon, 0<\epsilon<\frac{\pi}{2}$, such that

$$
\limsup _{r \rightarrow+\infty} \frac{\log n(r, \theta, \epsilon, f=a)}{\log \log r}=\lambda-1
$$


holds for each complex number $a$ (including $\infty$ ), with at most one possible exception? In section 9, we give some results about Borel directions for functions with finite logarithmic order, and we answer Wu's question in the negative in Theorem 9.3 .

\section{AN INTEGRAL CRITERION FOR A POSITIVE INCREASING FUNCTION TO BE OF FINITE LOGARITHMIC ORDER}

A positive increasing function $S(r)$, defined for $r>0$, is said to be of finite logarithmic order $\lambda$ if

$$
\limsup _{r \rightarrow+\infty} \frac{\log S(r)}{\log \log r}=\lambda
$$

$S(r)$ is said to be of infinite logarithmic order if the limit superior above is infinite.

Theorem 1.1. Let $S(r)$ be a positive increasing function defined for $r>0$. Then $S(r)$ is of logarithmic order $\lambda(0 \leq \lambda<+\infty)$ if and only if the integral

$$
\int^{\infty} \frac{S(r)}{r(\log r)^{\mu+1}} d r
$$

is convergent for $\mu>\lambda$ and divergent for $\mu<\lambda$.

To prove Theorem 1.1 we need a lemma as follows:

Lemma 1.1. If the integral (1.2) is convergent for $\mu>0$, then $S(r)$ is of logarithmic order not exceeding $\mu$.

Proof of Lemma 1.1. Since $S(r)$ is positive and increasing, we see that

$$
\begin{aligned}
\frac{S(r)}{\mu(\log r)^{\mu}} & =S(r) \int_{r}^{+\infty} \frac{1}{t(\log t)^{\mu+1}} d t \\
& <\int_{3}^{+\infty} \frac{S(r)}{r(\log r)^{\mu+1}} d r<+\infty
\end{aligned}
$$

holds for $r>3$ which implies that $S(r)$ is of logarithmic order not exceeding $\mu$.

Proof of Theorem 1.1. We prove this theorem in two steps.

Step I. Let $S(r)$ be of logarithmic order $\lambda$. For $\mu=\lambda+\epsilon(>\lambda)$, there exists a positive number $r_{\epsilon}$ such that $S(r)<(\log r)^{\lambda+\frac{\epsilon}{2}}$ for $r>r_{\epsilon}$. It follows that

$$
\begin{aligned}
\int^{+\infty} \frac{S(r)}{r(\log r)^{\mu+1}} d r & =\int^{r_{\epsilon}} \frac{S(r)}{r(\log r)^{\mu+1}} d r+\int_{r_{\epsilon}}^{+\infty} \frac{S(r)}{r(\log r)^{\mu+1}} d r \\
& <O(1)+\int_{r_{\epsilon}}^{+\infty} \frac{1}{r(\log r)^{1+\epsilon / 2}} d r \\
& <+\infty .
\end{aligned}
$$

On the other hand, for $\mu<\lambda$, the integral (1.2) is divergent. Indeed, if (1.2) is convergent, it follows from Lemma 1.1 that $S(r)$ is of logarithmic order $\leq \mu<\lambda$. This contradicts the fact that $S(r)$ has logarithmic order $\lambda$. Therefore the integral (1.2) is convergent for $\mu>\lambda$ and divergent for $\mu<\lambda$.

Step II. We assume that the integral (1.2) is convergent for $\mu>\lambda$ and divergent for $\mu<\lambda$. By Lemma 1.1, the logarithmic order of $S(r) \leq \lambda+\epsilon$, for any positive number $\epsilon$, hence the logarithmic order of $S(r) \leq \lambda$. 
Suppose $S(r)$ has logarithmic order $\lambda-2 \epsilon$ for some positive $\epsilon$. By Step I, we see that the integral (1.2) is convergent for $\mu=(\lambda-2 \epsilon)+\epsilon=\lambda-\epsilon$. This contradicts the assumption of Step II. Hence the logarithmic order of $S(r)=\lambda$. Results in Step I and Step II complete the proof of Theorem 1.1.

The criterion in Theorem 1.1 is very useful for measuring the growth of any positive increasing function $S(r)$ defined for $r>0$; in particular, if $f(z)$ is a nonconstant meromorphic function, we can use $T(r, f), N(r, f=a)$ and $n(r, f=a)$ for $S(r)$. When $f(z)$ is an entire function, we can use $\log ^{+} M(r, f)$ for $S(r)$.

\section{THE LOGARITHMIC ORDER FOR MEROMORPHIC FUNCTIONS}

Definition. If $f(z)$ is a function meromorphic in the complex plane $\mathbb{C}$, the logarithmic order of $f$ is the logarithmic order of its characteristic function $T(r, f)$.

It is clear that the logarithmic order of a non-constant rational function is 1 . As we shall see in Theorem 7.3, there exist transcendental entire functions of logarithmic order 1 .

Let $M(r, f)$ be the maximum modulus of an entire function $f(z)$ over $|z| \leq r$. Since

$$
T(r, f) \leq \log ^{+} M(r, f) \leq((\rho+r) /(\rho-r)) T(\rho, f)
$$

for $0<r<\rho$ (cf. [Ne, p. 24]), $T(r, f)$ and $\log ^{+} M(r, f)$ are of the same logarithmic order, and hence the logarithmic order of $f(z)$ could also be defined by the number

$$
\limsup _{r \rightarrow+\infty} \frac{\log ^{+} \log ^{+} M(r, f)}{\log \log r} .
$$

Let $f(z)$ be a meromorphic function of finite positive logarithmic order $\lambda$. A non-negative continuous function $\lambda(r)$ defined in $(0,+\infty)$ is said to be a proximate logarithmic order of $T(r, f)$, if $\lambda(r)$ satisfies the following three conditions:

(1) $\lim _{r \rightarrow+\infty} \lambda(r)=\lambda$.

(2) $\lambda^{\prime}(r)$ exists everywhere in $(0,+\infty)$ except possibly in a countable set where $\lambda^{\prime}\left(r^{+}\right)$and $\lambda^{\prime}\left(r^{-}\right)$exist. Moreover, if we use the one-sided derivative $\lambda^{\prime}\left(r^{+}\right)$or $\lambda^{\prime}\left(r^{-}\right)$instead of $\lambda^{\prime}(r)$ of $r$ in the exceptional set, then

$$
\lim _{r \rightarrow+\infty} r(\log r) \lambda^{\prime}(r) \log \log r=0 .
$$

(3) Let $U(r, f)=(\log r)^{\lambda(r)}$. We have $T(r, f) \leq U(r, f)$ for sufficiently large $r$ and

$$
\limsup _{r \rightarrow+\infty} \frac{T(r, f)}{U(r, f)}=1
$$

The above function $U(r, f)$ is called a logarithmic type function of $T(r, f)$.

Theorem 2.1. If $f(z)$ is a meromorphic function of finite positive logarithmic order $\lambda$, then $T(r, f)$ has a proximate logarithmic order $\lambda(r)$.

Proof of Theorem 2.1. This theorem can be proven by using $\log r$ instead of $r$, and then by using the argument adopted by Yang in Theorem 6.1 of $\mathrm{Ya}$, p. 173]. We omit the details. 


\section{The LOGARITHMiC EXPONENT OF CONVERGENCE OF $a$-POINTS OF A FUNCTION}

Let $f(z)$ be a meromorphic function. For each $a \in \widehat{\mathbb{C}}=\mathbb{C} \cup\{\infty\}$, an $a$-point of $f(z)$ means a root of the equation $f(z)=a$. Let $\left\{z_{j}(a)\right\}$ be the sequence of $a$-points of $f(z)$ with $r_{j}(a) \leq r_{j+1}(a)$, where $r_{j}(a)=\left|z_{j}(a)\right|$. The logarithmic exponent of convergence of a-points of $f(z)$ is a number $\rho_{\log }(a)$ which is defined by

$$
\rho_{\log }(a)=\inf \left\{\left.\mu\left|\mu>0, \sum_{j} 1 /\right| \log r_{j}(a)\right|^{\mu}<+\infty\right\} .
$$

This quantity plays an important role in measuring the value distribution of $a$ points of $f(z)$.

Throughout this paper, we denote the logarithmic order of $n(r, f=a)$ by $\lambda_{\log }(a)$, where $n(r, f=a)$ is the number of roots of the equation $f(z)=a$ in $|z| \leq r$. It is well known that if a meromorphic function $f(z)$ is of finite order, then the order of $n(r, f=a)$ equals the exponent of convergence of $a$-points of $f(z)$. The following theorem is a corresponding result for meromorphic functions of finite logarithmic order.

Theorem 3.1. If $f(z)$ is a non-constant meromorphic function and of finite logarithmic order, then for each $a \in \widehat{\mathbb{C}}$, the logarithmic order of $n(r, f=a)$ equals the logarithmic exponent of convergence of a-points of $f(z)$.

Proof of Theorem 3.1. For each $\epsilon>0$, by the definition of $\lambda_{\log }(a)$, we see that

$$
j<\left(\log r_{j}(a)\right)^{\lambda_{\log }(a)+\epsilon}
$$

for sufficiently large values of $j$. If $\mu>\lambda_{\log }(a)$, take $\epsilon>0$ such that $\mu>\lambda_{\log }(a)+\epsilon$ and set $\mu_{1}=\mu /\left(\lambda_{\log }(a)+\epsilon\right)$. Then we get

$$
1 /\left|\log r_{j}(a)\right|^{\mu}<j^{-\mu_{1}}
$$

and hence

$$
\sum_{j} 1 /\left|\log r_{j}(a)\right|^{\mu}<\sum_{j} j^{-\mu_{1}}<+\infty .
$$

We deduce from these that

$$
\rho_{\log }(a) \leq \lambda_{\log }(a)
$$

If $\mu<\lambda_{\log }(a)$, there exists a sequence of positive integers $\left\{n_{j}\right\}$ such that

$$
b_{n_{j}}=1 /\left|\log r_{n_{j}}(a)\right|^{\mu}>1 / n_{j} .
$$

For $n_{j}>2$, letting $i_{j}$ denote the integral part of $n_{j} / 2$ and $b_{k}=1 /\left|\log r_{k}(a)\right|^{\mu}$, we have

$$
b_{i_{j}}+b_{i_{j}+1}+\cdots+b_{n_{j}}>\left(n_{j}-i_{j}+1\right) / n_{j}>1 / 2
$$

and hence

$$
\sum_{j} 1 /\left|\log r_{j}(a)\right|^{\mu}=+\infty
$$

Thus, we deduce that

$$
\lambda_{\log }(a) \leq \rho_{\log }(a) .
$$

The theorem follows from (3.5) and (3.9). 


\section{Properties of A meromorphic function of Finite logarithmic order}

First we prove a technical lemma:

Lemma 4.1. If $f(z)$ is a meromorphic function in $\mathbb{C}$, let $r_{j}(a)(j=1,2, \ldots)$ be the moduli of the a-points of $f(z)$, with $r_{j}(a) \leq r_{j+1}(a)$. If $\mu>0$, then the series

$$
\sum_{j} 1 /\left|\log r_{j}(a)\right|^{\mu}
$$

and the integrals

$$
\int^{\infty} \frac{n(t, f=a)}{t(\log t)^{\mu+1}} d t
$$

and

$$
\int^{\infty} \frac{N(t, f=a)}{t(\log t)^{\mu+2}} d t
$$

are either simultaneously convergent or simultaneously divergent.

Proof of Lemma 4.1. From the identities

$$
\begin{aligned}
\sum_{j=j_{0}}^{J} \frac{1}{\left|\log r_{j}\right|^{\mu}} & =\int_{r_{0}}^{R} \frac{1}{|\log t|^{\mu}} d n(t, f=a) \\
& =\left[\frac{n(t, f=a)}{(\log t)^{\mu}}\right]_{t=r_{0}}^{t=R}+\mu \int_{r_{0}}^{R} \frac{n(t, f=a)}{t(\log t)^{\mu+1}} d t
\end{aligned}
$$

we deduce that the series (4.1) and the integral (4.2) are either simultaneously convergent or simultaneously divergent.

Next, by using the identity

$$
\int_{3}^{R} \frac{n(t, f=a)}{t(\log t)^{\mu+1}} d t=\left[N(t, f=a)(\log t)^{-\mu-1}\right]_{t=3}^{t=R}+(\mu+1) \int_{3}^{R} \frac{N(t, f=a)}{t(\log t)^{\mu+2}} d t
$$

we see that the integral (4.2) and the integral (4.3) are either simultaneously convergent or simultaneously divergent. This completes the proof of Lemma 4.1.

For the function $n(r, f=a)$ we have:

Lemma 4.2. If $n(r, f=a)$ is of finite positive logarithmic order, then we have

$$
\begin{array}{rlrl}
\sum_{j} 1 /\left|\log r_{j}(a)\right|^{\mu} & <+\infty, & \text { if } \mu>\lambda_{\log }(a) \\
& =+\infty, & & \text { if } \mu<\lambda_{\log }(a), \\
\int^{\infty} \frac{n(t, f=a)}{t(\log t)^{\mu+1}} d t & <+\infty, & \text { if } \mu>\lambda_{\log }(a) \\
& =+\infty, & \text { if } \mu<\lambda_{\log }(a), \\
\int^{\infty} \frac{N(t, f=a)}{t(\log t)^{\mu+2}} d t & & & \text { if } \mu>\lambda_{\log }(a) \\
& =+\infty, & & \text { if } \mu<\lambda_{\log }(a) .
\end{array}
$$

Proof of Lemma 4.2. By the definition of $\rho_{\log }(a)$, we have

$$
\begin{aligned}
\sum_{j} 1 /\left|\log r_{j}(a)\right|^{\mu} & <+\infty, \quad \text { if } \mu>\rho_{\log }(a) \\
= & +\infty, \quad \text { if } \mu<\rho_{\log }(a) .
\end{aligned}
$$


(4.6) follows from (4.9) and $\rho_{\log }(a)=\lambda_{\log }(a)$ (see Theorem 3.1); (4.7) follows from (4.6), because (by Lemma 4.1) the series (4.1) and the integral (4.2) are either simultaneously convergent or simultaneously divergent. Similarly (4.8) follows from (4.6), since the series (4.1) and the integral (4.3) are either simultaneously convergent or simultaneously divergent. This completes the proof of Lemma 4.2.

Although for any given meromorphic function $f(z)$ with finite positive order and for any $a \in \widehat{\mathbb{C}}$, the counting functions $N(r, f=a)$ and $n(r, f=a)$ both have the same order, the situation is different for functions of finite logarithmic order. Indeed, we have the following.

Theorem 4.1. Let $f(z)$ be a non-constant meromorphic function in $\mathbb{C}$. For each $a \in \widehat{\mathbb{C}}, N(r, f=a)$ is of logarithmic order $\lambda_{\log }(a)+1$, where $\lambda_{\log }(a)$ is the logarithmic order of $n(r, f=a)$.

Proof of Theorem 4.1. The expression (4.8) and Theorem 1.1 imply that $N(r, f=a)$ is of logarithmic order $\lambda_{\log }(a)+1$, since $N(r, f=a)$ is a positive increasing function for $r>0$.

\section{ON LOGARITHMiC BOREL EXCEPTIONAL VALUES OF MEROMORPHIC FUNCTIONS WITH FINITE LOGARITHMIC ORDER}

According to Nevanlinna's Theory, a value $\alpha \in \widehat{\mathbb{C}}$ is called a Borel exceptional value of $f(z)$ if the order of $n(r, f=\alpha)$ is less than the order of $f(z)$, i.e., the inequality

$$
\limsup _{r \rightarrow+\infty} \frac{\log n(r, f=\alpha)}{\log r}<\lambda
$$

holds for $\alpha$, where $\lambda$ is the order of $f(z)$.

For a meromorphic function of zero order, then the order of $n(r, f=a)$ equals zero for each $a \in \widehat{\mathbb{C}}$. Cartwright [Ca, p. 24] wrote: the definition of Borel exceptional values fails for functions of zero order. However, in terms of functions of finite logarithmic order, Borel exceptional value can be defined as follows:

Definition. If $f(z)$ is a meromorphic function in $\widehat{\mathbb{C}}$ with finite logarithmic order $\lambda$, a complex number $\alpha$ is called a logarithmic Borel exceptional value of $f(z)$, if

$$
\lambda_{\log }(\alpha)=\limsup _{r \rightarrow+\infty} \frac{\log n(r, f=\alpha)}{\log \log r}<\lambda-1 .
$$

Furthermore, $\alpha$ is called a reduced logarithmic Borel exceptional value of $f(z)$ if

$$
\bar{\lambda}_{\log }(\alpha)=\limsup _{r \rightarrow+\infty} \frac{\log \bar{n}(r, f=\alpha)}{\log \log r}<\lambda-1,
$$

where $\bar{n}(r, f=\alpha)$ denotes the distinct roots of the equation $f(z)=\alpha$ in $|z| \leq r$.

Corresponding to the classical result of Borel, we have, for meromorphic functions of finite logarithmic order, the following theorem.

Theorem 5.1 (The Logarithmic Borel Exceptional Value Theorem). If $f(z)$ is a non-constant meromorphic function of finite logarithmic order in $\mathbb{C}$, then $f(z)$ has at most two logarithmic Borel exceptional values. 
Proof of Theorem 5.1. Case (1). If $f(z)$ is a non-constant rational function, then $f(z)$ has logarithmic order 1 and $\lambda_{\log }(a)=0$ for each $a \in \widehat{\mathbb{C}}$. Thus, the conclusion of the theorem follows immediately.

Case (2). When $f(z)$ is transcendental and of finite logarithmic order $\lambda$, suppose there exist three distinct logarithmic Borel exceptional values $a_{i}(i=1,2,3)$. Then

$$
\limsup _{r \rightarrow+\infty} \frac{\log n\left(r, f=a_{i}\right)}{\log \log r}<\lambda-1 .
$$

By (5.4) and Theorem 4.1 we have

$$
\limsup _{r \rightarrow+\infty} \frac{\log N\left(r, f=a_{i}\right)}{\log \log r}<\lambda
$$

for $i=1,2,3$. We may assume, without loss of generality, that $a_{1}=0, a_{2}=1, a_{3}=$ $\infty$, and $f(0) \neq 0,1, \infty$ and $f^{\prime}(0) \neq \infty$. The original second fundamental theorem of R. Nevanlinna (see [Ya, p. 14, Theorem 1.4]) asserts that

$$
\begin{aligned}
T(r, f) \leq & \sum_{i=1}^{3} N\left(r, f=a_{i}\right)-\left\{2 N(r, f=\infty)-N\left(r, f^{\prime}=\infty\right)\right. \\
& \left.+N\left(r, f^{\prime}=0\right)\right\}+S(r, f) \\
\leq & \sum_{i=1}^{3} N\left(r, f=a_{i}\right)+S(r, f),
\end{aligned}
$$

where

$$
S(r, f)=m\left(r, \frac{f^{\prime}}{f}\right)+m\left(r, \frac{f^{\prime}}{f-1}\right)+\log \left|\frac{f(0)(f(0)-1)}{f^{\prime}(0)}\right|+\log 2 .
$$

By the lemma on logarithmic derivatives (see [Ya, p. 17, Lemma 1.3]), we have

$$
S(r, f)=O(\log r)+4 \log ^{+} T(\rho, f),
$$

where $0<r<\rho$, for sufficiently large $r$. Since $f(z)$ is a transcendental meromorphic function of finite logarithmic order, (5.8) implies that

$$
S(r, f)=O(\log r)=o(T(r, f)) .
$$

Now (5.6) and (5.9) imply that

$$
(1-o(1)) T(r, f)<\sum_{i=1}^{3} N\left(r, f=a_{i}\right)
$$

for sufficiently large $r$.

By (5.5) and (5.10), we have

$$
\limsup _{r \rightarrow+\infty} \frac{\log T(r, f)}{\log \log r}<\lambda .
$$

This contradicts the fact that $T(r, f)$ has logarithmic order $\lambda$. This completes the proof of Theorem 5.1.

From Theorem 5.1 and the fact that $N(r, f=a)$ is of logarithmic order $\lambda_{\log }(a)+$ 1 , we have the following corollary. 
Corollary 5.1. If $f(z)$ is a non-constant meromorphic function in $\mathbb{C}$ with finite logarithmic order $\lambda$, then for each $a \in \widehat{\mathbb{C}}$, the equation

$$
\lambda_{\log }(a)=\lambda-1
$$

holds with at most two possible exceptional values of a.

Corollary 5.1 can be viewed as a quantitive version of Theorem 5.1.

\section{RESUlts CONCERNING DERIVATIVES OF MEROMORPHIC FUNCTIONS}

Let $\lambda_{\log }(f)$ denote the logarithmic order of $T(r, f)$.

Theorem 6.1. If $f(z)$ is a transcendental meromorphic function in $\mathbb{C}$ with finite logarithmic order, then $f(z)$ and its derivative $f^{\prime}(z)$ have the same logarithmic order.

Proof of Theorem 6.1. Since $T\left(r, f^{\prime}\right)=m\left(r, f^{\prime}\right)+N\left(r, f^{\prime}\right) \leq 2 T(r, f)+m\left(r, f^{\prime} / f\right)$ and $m\left(r, f^{\prime} / f\right)=O(\log r)$, as $r \rightarrow+\infty$, we have $\lambda_{\log }\left(f^{\prime}\right) \leq \lambda_{\log }(f)$.

On the other hand, applying an inequality of Chuang Chi-tai [Ch], Ya, p. 95, Theorem 4.1], we have $\lambda_{\log }(f) \leq \lambda_{\log }\left(f^{\prime}\right)$. This completes the proof of Theorem 6.1 .

Theorem 6.2. If $f(z)$ is a transcendental meromorphic function in $\mathbb{C}$ with finite logarithmic order $\lambda$, then we have

$$
\limsup _{r \rightarrow+\infty} \frac{\log \left\{n(r, f=0)+\bar{n}\left(r, f^{(k)}=1\right)\right\}}{\log \log r}=\lambda-1,
$$

for each positive integer $k$.

To prove Theorem 6.2 we need the following two results.

Lemma 6.1 (An inequality on the logarithmic derivative of K. L. Hiong (see Hi], Ya, p. 99, Lemma 4.3]). Let $f(z)$ be a non-constant meromorphic function in $\mathbb{C}$. If $f(0) \neq 0, \infty$, then for every positive integer $k$,

$m\left(r, \frac{f^{(k)}}{f}\right)<C_{k}\left\{1+\log ^{+} \log ^{+} \frac{1}{|f(0)|}+\log ^{+} \frac{1}{r}+\log ^{+} \frac{1}{\rho-r}+\log ^{+} \rho+\log ^{+} T(\rho, f)\right\}$,

where $0<r<\rho<R$ and $C_{k}$ is a constant depending only on $k$.

Lemma 6.2 (An inequality of Hayman (see [Ha1, Ya, p. 110, 4.5]). Let $f(z)$ be a transcendental meromorphic function in $\mathbb{C}$ and let $k$ be a positive integer. If $f(0) \neq 0, \infty, f^{(k)}(0) \neq 1, f^{(k+1)}(0) \neq 0$ and

$$
(k+1) f^{(k+2)}(0)\left(f^{(k)}(0)-1\right)-(k+2) f^{(k+1)}(0)^{2} \neq 0,
$$

then

$$
T(r, f)<\left(2+\frac{1}{k}\right) N(r, f=0)+\left(2+\frac{2}{k}\right) \bar{N}\left(r, f^{(k)}=1\right)+S^{*}(r, f),
$$


for $0<r<R$, where

$$
\begin{aligned}
S^{*}(r, f)=(2+ & \left.\frac{2}{k}\right) m\left(r, \frac{f^{(k+1)}}{f^{(k)}-1}\right)+\left(2+\frac{1}{k}\right)\left\{m\left(r, \frac{f^{(k+1)}}{f}\right)+m\left(r, \frac{f^{(k)}}{f}\right)\right\} \\
& +\frac{1}{k} m\left(r, \frac{f^{(k+2)}}{f^{(k+1)}}\right)+4+\left(2+\frac{1}{k}\right) \log \left|\frac{f(0)\left(f^{(k)}(0)-1\right)}{f^{(k+1)}(0)}\right| \\
& +\frac{1}{k} \log \left|\frac{f^{(k+1)}(0)\left(f^{(k)}(0)-1\right)}{(k+1) f^{(k+2)}(0)\left(f^{(k)}(0)-1\right)-(k+2) f^{(k+1)}(0)^{2}}\right| .
\end{aligned}
$$

Proof of Theorem 6.2. Since $f(z)$ is of finite logarithmic order $\lambda$, by Theorem 4.1 and Theorem 6.1, $n(r, f=0)+\bar{n}\left(r, f^{(k)}=1\right)$ has logarithmic order $\leq \lambda-1$. Hence, to prove (6.1), it suffices to show that the inequality

$$
\limsup _{r \rightarrow+\infty} \frac{\log \left\{n(r, f=0)+\bar{n}\left(r, f^{(k)}=1\right)\right\}}{\log \log r} \geq \lambda-1
$$

holds.

Suppose the inequality (6.5) does not hold. Then $n(r, f=0)+\bar{n}\left(r, f^{(k)}=1\right)$ has logarithmic order less than $\lambda-1$.

By using Lemma 6.1 and Lemma 6.2, for any fixed $\tau>1$, we have

$$
S^{*}(r, f)=O\left(\log ^{+} T(\tau r, f)\right)+O(\log r) \quad \text { as } \quad r \rightarrow+\infty .
$$

By (6.6) and a result of [Ya, p. 25, Lemma 1.5], we deduce that

$$
S^{*}(r, f)=O(\log r)=o(T(r, f)) .
$$

Therefore, by (6.3), $T(r, f)$ has logarithmic order less than $\lambda$. This contradicts the fact that $T(r, f)$ has logarithmic order $\lambda$. Hence inequality (6.5) holds. This completes the proof of Theorem 6.2.

The following Theorem 6.3 follows immediately from Theorem 6.2.

Theorem 6.3. If $f$ is a transcendental meromorphic function in $\mathbb{C}$ with finite logarithmic order, then the following two cases cannot occur simultaneously:

(i) $f(z)$ has a finite Borel exceptional value,

(ii) $f^{(k)}(z)$ has a finite non-zero reduced Borel exceptional value for a positive integer $k$.

\section{Growth dominated By two values}

For a transcendental meromorphic function $f(z), T(r, f)$ is usually dominated by three integrated counting functions. However, when $f(z)$ is of finite logarithmic order, $T(r, f)$ can be dominated by two integrated counting functions as the following shows:

Theorem 7.1. If $f(z)$ is a transcendental meromorphic function of finite logarithmic order $\lambda$, then for any two distinct extended complex values a and $b$, we have

$$
T(r, f) \leq N(r, f=a)+N(r, f=b)+o(U(r, f)),
$$


where $U(r, f)=(\log r)^{\lambda(r)}$ is a logarithmic-type function of $T(r, f)$. Furthermore, if $T(r, f)$ has finite lower logarithmic order

$$
\mu=\liminf _{r \rightarrow+\infty} \frac{\log T(r, f)}{\log \log r},
$$

with $\lambda-\mu<1$, then

$$
T(r, f) \leq N(r, f=a)+N(r, f=b)+o(T(r, f)) .
$$

Proof of Theorem 7.1. For any two distinct complex numbers $a$ and $b$ which are neither zero nor infinity, we have

$$
T\left(r, \frac{f-a}{f-b}\right)=T(r, f)+O(1) .
$$

Without loss of generality, we may assume that $a=0, b=\infty$ and $f(0) \neq 0, \infty$. Put

$$
f(z)=\frac{P_{1}(z)}{P_{2}(z)}
$$

where $P_{1}(z)$ and $P_{2}(z)$ are the canonical products formed from the zeros and poles of $f(z)$, respectively.

Since $f(z)$ has logarithmic order $\lambda$, and $n(r, f=0)$ and $n(r, f=\infty)$ both have logarithmic order $\leq \lambda-1$, we get the following inequality via integration by parts:

$T(r, f) \leq \log M\left(r, P_{1}\right)+\log M\left(r, P_{2}\right)$

$$
\leq \int_{0}^{r} \frac{n(t, f=0)+n(t, f=\infty)}{t} d t+r \int_{r}^{+\infty} \frac{n(t, f=0)+n(t, f=\infty)}{t^{2}} d t
$$

$$
\leq N(r, f=0)+N(r, f=\infty)+r \int_{r}^{+\infty} \frac{(\log t)^{\lambda-1+\epsilon}}{t^{2}} d t
$$

for any small positive number $\epsilon$, when $r$ is sufficiently large. Hence

$$
\begin{aligned}
T(r, f) & \leq N(r, f=0)+N(r, f=\infty)+\left[(\log r)^{\lambda-1+\epsilon}+\cdots+O(\log r)\right] \\
& =N(r, f=0)+N(r, f=\infty)+o(U(r, f)) .
\end{aligned}
$$

If $\lambda-\mu<1$, take $\epsilon>0$ such that $\lambda-\mu<1-\epsilon$. Applying (7.7), we have

$$
T(r, f) \leq N(r, f=0)+N(r, f=\infty)+o(T(r, f)) .
$$

This completes the proof of Theorem 7.1.

As an application of Theorem 7.1, we have a result sharper than Theorem 5.1 in the sense that the two possible logarithmic Borel exceptional values can be replaced by one possible logarithmic Borel exceptional value as follows.

Theorem 7.2. If $f(z)$ is a non-constant meromorphic function with finite logarithmic order $\lambda$, then $f(z)$ has at most one logarithmic Borel exceptional value. In particular, if $f(z)$ is an entire function of finite logarithmic order, then $f(z)$ has no finite logarithmic Borel exceptional values.

Theorem 7.2 is an immediate consequence of Theorem 7.1.

Theorem 7.3. There are infinitely many transcendental entire functions of logarithmic order one. 
Proof of Theorem 7.3. For each positive number $c>1$, if we put

$$
g_{c}(z)=\prod_{n=1}^{+\infty}\left(1-\frac{z}{e^{c^{n}}}\right)
$$

then we have

$$
\rho_{\log }\left(g_{c}=0\right)=\inf \left\{\mu \mid \mu>0, \sum_{n=1}^{+\infty} \frac{1}{\left(c^{n}\right)^{\mu}}<+\infty\right\}=0 .
$$

It follows that $n\left(r, g_{c}=0\right)$ has logarithmic order zero, since $\lambda_{\log }(0)=\rho_{\log }(0)$ by Theorem 3.1. By Theorem 4.1, $N\left(r, g_{c}=0\right)$ has logarithmic order one. $g_{c}(z)$ is an entire function and is a canonical product formed with zeros of $g_{c}(z)$, and it follows from the expression (7.3) of Theorem 7.1 that $T\left(r, g_{c}\right)$ has logarithmic order one. Since $c>1$ is arbitrary, the proof of Theorem 7.3 is complete.

Theorem 7.4. For each positive number $\lambda>1$, there is an entire function of logarithmic order $\lambda$.

Proof of Theorem 7.4. Put $k=\lambda-1(>0)$, and

$$
f_{k}(z)=\prod_{n=1}^{+\infty}\left(1-\frac{z}{e^{n^{1 / k}}}\right)
$$

Then

$$
\rho_{\log }\left(f_{k}=0\right)=\inf \left\{\mu \mid \mu>0, \sum_{n=1}^{+\infty} \frac{1}{\left(n^{1 / k}\right)^{\mu}}<+\infty\right\}=k .
$$

It follows that $n\left(r, f_{k}=0\right)$ has logarithmic order $k$, since

$$
\lambda_{\log }\left(f_{k}=0\right)=\rho_{\log }\left(f_{k}=0\right) .
$$

By Theorem 4.1, $N\left(r, f_{k}=0\right)$ has logarithmic order $k+1$. Note that $f_{k}(z)$ is an entire function and is a canonical product formed with its zeros. It follows from the expression (7.3) of Theorem 7.1 that $T\left(r, f_{k}\right)$ has logarithmic order $k+1=\lambda$. This completes the proof of Theorem 7.4.

\section{Asymptotic BehaViors on Value distribution of ENTIRE FUnCtions WITH FINITE LOGARITHMIC ORDER}

Let $a(r), b(r)$ be two positive functions defined for $r>0$. If $\lim _{r \rightarrow+\infty} a(r) / b(r)$ $=1$, we write $a(r) \sim b(r)$.

In 1929, G. Valiron and E. F. Collingwood [VC] proved that if $f(z)$ is a transcendental entire function satisfying

$$
\log M(r, f)=O\left((\log r)^{2}\right),
$$

then for every $a \in \mathbb{C}$

$$
\log M(r, f) \sim N(r, f=a) .
$$

We have the following related results.

Theorem 8.1. Let $f(z)$ be a transcendental entire function with finite logarithmic order $\lambda$ and lower logarithmic order $\mu\left(=\liminf _{r \rightarrow+\infty} \frac{\log T(r, f)}{\log \log r}\right)$. If $\lambda-\mu<1$, then for every finite complex value a, we have

$$
N(r, f=a) \sim T(r, f) \sim \log M(r, f) .
$$


Theorem 8.1 is an extension of the result of Valiron and Collingwood mentioned above. It is an immediate consequence of the next theorem.

Theorem 8.2. Let $m$ and $c$ be non-negative numbers satisfying $0 \leq c<1$ and $m-1>c$, and let $f(z)$ be a transcendental entire function of finite logarithmic order satisfying both conditions

$$
\log M(r, f)=O\left((\log r)^{m-c}\right)
$$

and

$$
\liminf _{r \rightarrow+\infty} \frac{\log M(r, f)}{(\log r)^{m-1}}=+\infty .
$$

Then for every finite complex value a, we have

$$
N(r, f=a) \sim T(r, f) \sim \log M\left(r(\log r)^{c}, f\right) .
$$

The case $m=2$ in the above theorem was due to Q. I. Rahman [Ra] in 1957.

Proof of Theorem 8.2. Without loss of generality, we may assume $f(0) \neq a$. Jensen's Theorem says that

$$
N(r, f=a)=\frac{1}{2 \pi} \int_{0}^{2 \pi} \log \left|f\left(r e^{i \theta}\right)-a\right| d \theta-\log |f(0)-a| .
$$

It follows from Jensen's Theorem that

$$
n(r, f=a)<\frac{1}{\log r}\left\{\log M\left(r^{2}, f-a\right)+O(1)\right\}=O\left((\log r)^{m-1-c}\right),
$$

by (8.3). Let $K$ be a positive number less than 1 . Now put $r=r_{1}\left(\log r_{1}\right)^{c}$ with $r_{1}>e^{(1-c) / K}$. Using (8.7) and the fact that $(\log r)^{m-1-c} / r^{K}$ is decreasing in $r$, we have the following:

$$
\begin{aligned}
\int_{r_{1}}^{+\infty} \frac{n(t, f=a)}{t^{2}} d t & =O\left(\int_{r_{1}}^{+\infty} \frac{1}{t^{2}}(\log t)^{m-1-c} d t\right) \\
& =O\left(\frac{\left(\log r_{1}\right)^{m-1-c}}{r_{1}^{K}} \int_{r_{1}}^{+\infty} \frac{1}{t^{2-K}} d t\right) \\
& =O\left(\frac{\left(\log r_{1}\right)^{m-1-c}}{r_{1}}\right) .
\end{aligned}
$$

Therefore we see that

$$
r_{1} \int_{r_{1}}^{+\infty} \frac{n(t, f=a)}{t^{2}} d t=O\left(\left(\log r_{1}\right)^{m-1-c}\right) .
$$

Since $f(z)-a$ is of genus zero, we have

$$
\begin{aligned}
\log M(r, f) & <\int_{0}^{+\infty} \frac{r n(t, f=a)}{t(t+r)} d t+O(\log r) \\
& \leq \int_{0}^{r_{1}} \frac{r n(t, f=a)}{t(t+r)} d t+r \int_{r_{1}}^{+\infty} \frac{n(t, f=a)}{t^{2}} d t+O(\log r) \\
& <N\left(r_{1}, f=a\right)+O\left(\left(\log r_{1}\right)^{m-1}\right)+O(\log r) .
\end{aligned}
$$


We deduce that

$$
(1-o(1)) \log M\left(r_{1}\left(\log r_{1}\right)^{c}, f\right)<N\left(r_{1}, f=a\right),
$$

which implies (8.5). This completes the proof of Theorem 8.2.

Theorem 8.2 has the following immediate consequence.

Corollary 8.1. If $f(z)$ is given as in Theorem 8.2, then

$$
\begin{aligned}
& N(r, f=a) \sim N\left(r(\log r)^{c}, f\right) \sim T(r, f) \sim T\left(r(\log r)^{c}, f\right) \\
& \quad \sim \log M(r, f) \sim \log M\left(r(\log r)^{c}, f\right), \quad \text { as } r \rightarrow+\infty .
\end{aligned}
$$

Theorem 8.3. If $f(z)$ is a transcendental entire function of finite logarithmic order $\lambda$ satisfying

$$
\log M(r, f)=O\left(r^{\delta(r)}(\log r)^{\lambda}\right)
$$

and

$$
\liminf _{r \rightarrow+\infty} \frac{\log M(r, f)}{(\log r)^{\lambda-1}}=+\infty,
$$

where $\delta(r)$ is a real decreasing function tending to zero as $r \rightarrow+\infty$ with $r^{\delta(r)}=$ $o(\log r)$, then for every $a \in \mathbb{C}$,

$$
\liminf _{r \rightarrow+\infty} \frac{N(r, f=a)}{\log M\left(r^{1-2 \delta\left(r^{2}\right)}, f\right)} \geq 1 .
$$

The case $\lambda=2$ in Theorem 8.3 was due to G. Valiron and E. F. Collingwood VC] in 1929.

Proof of Theorem 8.3. It follows from Jensen's Theorem and (8.13) that

$$
n(r, f=a)<\frac{\log M\left(r^{2}, f=a\right)}{\log r}=O\left(\left(r^{2}\right)^{\delta\left(r^{2}\right)}(\log r)^{\lambda-1}\right) .
$$

Now put $r=r_{1}^{1-2 \delta\left(r_{1}^{2}\right)}$. By (8.16) we have

$$
\begin{aligned}
\int_{r_{1}}^{+\infty} \frac{n(t, f=a)}{t^{2}} d t & =O\left(\int_{r_{1}}^{+\infty}(\log t)^{\lambda-1} t^{2 \delta\left(t^{2}\right)-2} d t\right) \\
& =O\left(\int_{r_{1}}^{+\infty} t^{(\lambda-1)(\log \log t / \log t)+2 \delta\left(t^{2}\right)-2} d t\right) \\
& =O\left(\int_{r_{1}}^{+\infty} t^{(\lambda-1)\left(\log \log r_{1} / \log r_{1}\right)+2 \delta\left(r_{1}^{2}\right)-2} d t\right) .
\end{aligned}
$$

Since $\delta\left(t^{2}\right)$ is a decreasing function in $t$, the last expression of (8.17) is

$$
O\left(\frac{r_{1}^{(\lambda-1)\left(\log \log r_{1} / \log r_{1}\right)+2 \delta\left(r_{1}^{2}\right)-1}}{1-2 \delta\left(r_{1}^{2}\right)-(\lambda-1) \log \log r_{1} / \log r_{1}}\right) .
$$

Since $f(z)-a$ is of genus zero, by [Ti, p. 271] and (8.18), we deduce that

$$
\begin{aligned}
\log M(r, f) & <\int_{0}^{r_{1}} \frac{r n(t, f=a)}{t(t+r)} d t+r \int_{r_{1}}^{+\infty} \frac{n(t, f=a)}{t^{2}} d t+O(\log r) \\
& <N\left(r_{1}, f=a\right)+O\left(\left(\log r_{1}\right)^{\lambda-1}\right)+O(\log r) .
\end{aligned}
$$


Therefore we see that

$$
(1-o(1)) \log M\left(r_{1}^{1-2 \delta\left(r_{1}^{2}\right)}, f\right)<N\left(r_{1}, f=a\right) .
$$

This completes the proof of Theorem 8.3.

Remarks. 1. For entire functions of finite logarithmic order, there are many welldeveloped results. Let $f(z)$ be an entire function and $\mu(r, f)$ be the minimum of $|f(z)|$ on $|z|=r$. In 1979 A. A. Gol'dberg [Go proved the following conjecture by P. D. Barry [Ba]: If $\log M(r, f) /(\log r)^{2}$ has the upper limit $\sigma$, then the upper limit of $\mu(r, f) / M(r, f)$ is at least $C(\sigma)$. Here $C(\sigma)$ is a positive constant depending only on $\sigma$. Later P. C. Fenton $\mathrm{Fe} 2$ proved that the same conclusion of Barry's conjecture holds when the $\lim \sup _{r \rightarrow+\infty}$ in the hypothesis is replaced by liminf $r \rightarrow+\infty$, and he also obtained that $\mu(r, f) / M(r, f) \leq C(\sigma)$ for all $r \geq r_{0}$ implies

$$
\liminf _{r \rightarrow+\infty} \frac{\log M(r, f)-\sigma(\log r)^{2}}{\log r} \geq-2 \sigma \log \delta,
$$

where $\delta=\max \left(r_{0},\left|f^{\prime}(0)\right|\right)$.

2. On the well-established results about the asymptotic behavior of the real part of entire functions of finite logarithmic order, one might refer to results of C. C. Davis and P. C. Fenton DF, P. C. Fenton [Fe1 and W. K. Hayman Ha2].

\section{Results CONCERNing Borel DiRECTIONS}

Corresponding to Theorem 5.1, on the angular distribution theory, we have proved the following (cf. [C3, Theorem 2]).

Theorem 9.1 (The existence theorem of Borel direction). Let $f(z)$ be meromorphic in $\mathbb{C}$ with finite logarithmic order $\lambda$. If $f(z)$ satisfies the growth condition

$$
\limsup _{r \rightarrow+\infty} \frac{T(r, f)}{(\log r)^{2}}=+\infty
$$

then there exists a direction $\Delta(\theta)=\{z: \arg z=\theta\}$ with $0 \leq \theta<2 \pi$ such that for each small positive number $\epsilon, 0<\epsilon<\pi / 2$, and every $a \in \widehat{\mathbb{C}}$, the equation

$$
\limsup _{r \rightarrow+\infty} \frac{\log n(r, \theta, \epsilon, f=a)}{\log \log r}=\lambda-1
$$

holds with at most two possible exceptional values of a, where $n(r, \theta, \epsilon, f=a)$ denotes the number of roots (multiple roots being counted with their multiplicities) of the equation $f(z)=a$ for $z$ in the angular region $\Omega(r, \theta, \epsilon)=\{z:|\arg z-\theta|<$ $\epsilon,|z|<r\}$.

The result is sharp. Indeed, A. Ostrowski Os constructed a transcendental meromorphic function $f(z)$ such that $T(r, f)=O\left((\log r)^{2}\right)$ which has no Julia direction. The ray $\Delta(\theta)$ in the above theorem is called a Borel direction of logarithmic order $\lambda-1$ for $f(z)$.

Theorem 9.2 (see [C3, Theorem 1]). There exists a meromorphic function $f(z)$ of logarithmic order 3 such that $f(z)$ and $f^{\prime}(z)$ have no common Borel direction (of logarithmic order 2). 
Motivated by Theorem 9.1 and Theorem 7.1, in a private communication, Seng Jian $\mathrm{Wu}$ (Beijing University, China) asked the following question:

Let $f(z)$ be given as in Theorem 9.1. Does there exist a ray $\Delta(\theta)$ such that for any small positive number $\epsilon, 0<\epsilon<\pi / 2$, and for every $a \in \widehat{\mathbb{C}}$, equation (9.2) holds with at most one possible exceptional value $a \in \widehat{\mathbb{C}}$ ?

Theorem 9.3. The answer to the above question of J. S. Wu is negative.

Proof of Theorem 9.3. Let

$$
f(z)=\prod_{n=1}^{+\infty} \frac{z+e^{\sqrt{n}}}{z-e^{\sqrt{n}}}
$$

be a canonical product from pole set $\left\{e^{\sqrt{n}}\right\}_{n=1}^{+\infty}$ and zero set $\left\{-e^{\sqrt{n}}\right\}_{n=1}^{+\infty}$. Since

$$
\rho_{\log }(f=0)=\rho_{\log }(f=\infty)=\inf \left\{\mu \mid \mu>0, \sum_{n=1}^{+\infty} \frac{1}{n^{\mu / 2}}<+\infty\right\}=2
$$

it follows from Theorem 3.1 that $n(r, f=0)$ and $n(r, f=\infty)$ both have logarithmic order 2 and since $f$ is a canonical product from poles set $\left\{e^{\sqrt{n}}\right\}_{n=1}^{+\infty}$ and zero set $\left\{-e^{\sqrt{n}}\right\}_{n=1}^{+\infty}$, it follows from Theorem 4.1 and Theorem 7.1 that $T(r, f)$ has logarithmic order 3 and satisfies the growth condition (9.1).

Now we assume that there is a ray $\Delta\left(\theta_{0}\right)$ which meets the requirement of the question of J. S. Wu. We put $\Omega_{1}=\left\{z: \frac{\pi}{2}-\epsilon<\arg z<\frac{\pi}{2}+\epsilon\right\}, \Omega_{2}=\left\{z: \frac{3 \pi}{2}-\epsilon<\right.$ $\left.\arg z<\frac{3 \pi}{2}+\epsilon\right\}, \Omega_{3}=\left\{z: \frac{\pi}{2}+\epsilon \leq \arg z \leq \frac{3}{2} \pi-\epsilon\right\}$, and $\Omega_{4}=\mathbb{C} \backslash \bigcup_{i=1}^{3} \Omega_{i}$. It is known (see [Ro]) that $f(z) \rightarrow 0$ uniformly for $z \in \Omega_{3}$ and $f(z) \rightarrow \infty$ uniformly for $z \in \Omega_{4}$. Hence $\Delta\left(\theta_{0}\right) \not \subset \Omega_{3} \cup \Omega_{4}$. In $\Omega_{1} \cup \Omega_{2}, f(z)$ omits zero and $\infty$. It follows that $\Delta\left(\theta_{0}\right) \not \subset \Omega_{1} \cup \Omega_{2}$. Hence $\Delta\left(\theta_{0}\right) \not \subset \mathbb{C}$, which contradicts our assumption. This completes the proof of Theorem 9.3.

Remark. For a transcendental meromorphic function $f(z)$, its Nevanlinna characteristic function $T(r, f)$ is usually dominated by the sum of the growth of three integrated counting functions. We obtain that when $f(z)$ is of finite logarithmic order, $T(r, f)$ can be dominated by the sum of the growth of two integral counting functions (see Theorem 7.1). However, the above Theorem 9.3 shows that it is impossible that the growth of a meromorphic functions of finite logarithmic order is always dominated by the sum of the growth of two distinct complex values, assumed by the function in an angular region which includes the Borel direction of finite logarithmic order.

\section{REFERENCES}

[Ba] P.D. Barry, The minimum modulus of small integral and subharmonic functions, Proc. London Math. Soc., (3) 12 (1962), 445-495. MR0139741 (25:3172)

[Ca] M.L. Cartwright, Integral Functions, Cambridge University Press, 1952. MR0077622 $(17: 1067 \mathrm{c})$

[Ch] C. Chuang, Sur la comparison de la croissance d'une fonction méromorphe et de celle de sa dérivée, Bull. Sci. Math. 75 (1951), 171-190. MR0045822 (13:640g)

[C1] T.Y. Peter Chern, Value distribution of meromorphic function with zero order (preprint, addressed in the Hedberg Conference, June, 1996, Linköping, Sweden.)

[C2] T.Y. Peter Chern, On the maximum modulus and the zeros of an transcendental entire function of finite logarithmic order, Bull. Hong Kong Math. Soc. 2 (1999), 271-278. MR1692738 (2000b:30034) 
[C3] T.Y. Peter Chern, Common Borel directions of meromorphic function with zero order and its derivative, Proc. Amer. Math. Soc. 132 (2004), no. 4, 1171-1175. MR2045434(2004k:30059)

[DF] C.C. Davis and P.C. Fenton, The real part of entire functions, Michigan Math. J. 43 (1996), no. 3, 475-494. MR1420588 (97j:30009)

[Fe1] P.C. Fenton, Wiman-Valiron theory for entire functions of finite lower growth, Trans. Amer. Math. Soc. 252 (1979), 221-232. MR0534119 (80i:30042)

[Fe2] P.C. Fenton, The minimum modulus of certain small entire functions, Trans. Amer. Math. Soc. 271 (1982), no. 1, 183-195. MR0648085 (83h:30022)

[Go] A.A. Gol'dberg, The minimum modulus of a meromorphic function of slow growth (Russian) Mat. Zametki 25 (1979), no. 6, 835-844, 956. MR0540239 (81c:30058)

[Ha1] W.K. Hayman, Picard values of meromorphic functions and their derivatives, Ann. of Math. 70 (1959), 9-42. MR0110807 (22:1675)

[Ha2] W.K. Hayman, The local growth of power series: a survey of the Wiman-Valiron method, Canad. Math. Bull. 17 (1974), no. 3, 317-358. MR0385095 (52:5965)

[Hi] K. Hiong, Sur les fonctions holomorphes dont les dérivées admettent une valeur exceptionnelle, Ann. École Norm. Sup. 72 (1955), 165-197. MR0074525 (17:600h)

[Ne] R. Nevanlinna, Le Theorems de Picard-Borel et la Theorie des Fonctions Meromorphes, Coll. Borel, 1929.

[Os] A. Ostrowski, Über Folgen analytischer Funktionen und einige Verschärfungen des Picardschen Satzes, Math. Zeit. 24 (1926), 215-258.

[Ra] Q.I. Rahman, On a class of integral functions of zero order, J. Lond. Math. Soc. 32 (1957), 109-110. MR0083037 (18:648c)

[Ro] J. Rossi, A sharp result concerning cercles de remplissage, Ann. Acad. Sci. Fenn. Ser. A I. Math., vol. 20 (1995), 179-185. MR1304116 (95k:30062)

[Su] J. Sun, On the characteristic of composite entire functions, J. East China Norm. Univ. Natur. Sci. Ed., 1998, no. 4, 29-36. MR1695896 (2000e:30055)

[Ti] E.C. Titchmarsh, Theory of Functions, Oxford University Press, New York, 1939.

[VC] G. Valiron and E.F. Collingwood, A theorem concerning integral functions of order less than 1, J. Lond. Math. Soc. 4 (1929), 210-213.

[Ya] L. Yang, Value Distribution Theory, Science Press (English Edition), Springer-Verlag, 1993. MR1301781 (95h:30039)

Department of Mathematics, Michigan State University, East Lansing, Michigan 48824

E-mail address: pchern@math.msu.edu

Current address: Department of Applied Mathematics, I-Shou University, Kaohsiung, Taiwan 840, R.O.C.

E-mail address: tychern@isu.edu.tw 